\title{
General Atmospheric Circulation of the Tropics
}

\author{
By \\ Herbert Riehl \\ Department of Atmospheric Science \\ Colorado State University \\ Fort Collins, Colorado
}

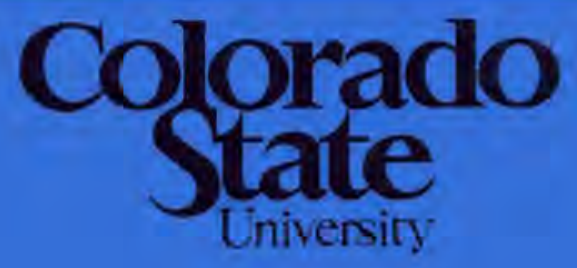

\section{Department of Atmospheric Science}




\section{CURRENT PROBLEMS IN RESEARCH}

The General Atmospheric Circulation of the Tropics

by

Herbert Riehl

Colorado State University

In science, we are always interested in a well-ordered simple package whenever such a package appears to be in the offing. There has been widespread belief that the general circulation of the tropics meets these requirements. The weather observations which have been gathered in increasing volume in the upper air over the tropics during the last 15 years, however, have thrown doubt on the validity of such a simple view. They appear to call for a more complex approach to an ultimate understanding of the tropical atmospheric machinery and of the interaction between tropical and temperate latitudes. The question now is: must we really accept an increased order of difficulty, or can the evidence of the new observations be reconciled with the older approach? If so, chances at arriving at a definite solution of the tropical general circulation problem within the next decade would be greatly enhanced.

In one respect the story has not changed; the tropics are a heat source for the atmosphere of higher latitudes. This fact, in broad terms, has been appreciated for centuries. Further, circumnavigation of the oceans by sailing vessels led to recognition of a second fact; the tropics also are the source of momentum for the westerly winds prevalent especially in temperate latitudes. Essentially half of the globe has winds from east at the surface (fig. 1), and the other 
half has winds from the west. Surface drag acts to retard all winds and bring air toward stagnation. Therefore, rnomentum in the direction of rotation of the earth's axis (toward east) is given up by the atmosphere to the earth outside of the tropics and taken up from earth inside the tropics. For balance, momentum must flow poleward across the subtropics.

Thus, we have a geophysical coincidence in the location of heat and momentum sources for the atmosphere. This fact must be rated as one of the basic peculiarities of our present-day planet, highly influential in governing life in the tropics. A second peculiarity, relevant here and to be explored, is that the surface of the tropics is mainly (about 80 per cent) oceanic.

\section{Simple Cell Model}

A realistic model of the tropical general circulation must do the following: (1) explain the principal observed circulation features and (2) permit export of heat and momentum to the northern and southern latitudes beyond. We can evolve a simple and very clear picture with three assumptions: (1) Steady state prevails except for diurnal and seasonal changes; (2) variations with longitude are small compared to those with latitude; (3) no cross-equator coupling exists again apart from seasonal changes and possible interaction on very long time scales (order of years and up). Assumption (1) will be supported by most travelers to the tropics; assumption (2) means that, while the strength of the trade winds may differ in Atlantic and Pacific Oceans, the significant point is that such a wind system exists in both oceans; assumption (3) rests on the fact that, broadly, the general circulation of both hemispheres is symmetrical with respect to the "meteorological equator" at $5^{\circ} \mathrm{N}$ latitude. 
Granted these assumptions, the general problem of the tropical general circulation involving time and three space coordinates as independent variables is reduced to two independent variables in space, latitude and height. We may then represent the salient features of the tropical atmosphere in vertical cross section (fig. 2).

The basic feature in fig. 2 is a "meridional circulation cell", with equatorward branch in the surface layer, return branch centered at 40,000 feet, ascending branch in the heart of the tropics and a descending branch -- not closed off from higher latitudes -. at the limit of the tropics. Warmest temperatures are situated near the equator from where they decrease poleward; near the surface cool air from outside the tropics is carried equatorward and warmed; in the return branch some heat is lost to space due to radiation.

The sense of the circulation is dictated by ascent where the air is warmest and least dense. Here the heat gained by the air over the oceans during equatorward passage -- largely through evaporation and manifest as an increase in moisture content of the atmosphere -- is released through precipitation in cumulus clouds. The other circulation branches follow from continuity. Subtropical deserts and equatorial rainbelt provide the first evidence for the existence and location of the vertical branches.

What can be said about the significance of the meridional cell?

(1) Despite the upward temperature decrease, the poleward branch carries more heat than the equatorward branch. The high altitude of the return flow must be taken into account. This produces potential energy transport poleward, and this transport is the dominating term in complete heat balance computations. 
(2) With ascent of light air near the equator and descent of heavier air on the margins of the tropics, the circulation acts to convert a small fraction of the potential energy, gained during the equatorial ascent, to energy of motion. This sustains the tropical wind system against frictional dissipation of kinetic energy.

(3) The air in the return branch, well removed from the surface, tends to conserve its angular momentum. The earth's angular momentum is highest at the equator where the distance from the earth's axis of rotation is greatest and where a point on the earth revolves at about $1000 \mathrm{mph}$. As the air travels toward higher latitudes, where the angular momentum of the earth becomes increasingly smaller, it should turn toward east. We should observe winds from west near 40, 000 feet, which rapidly increase in strength toward the poleward boundary of the cell. This is indeed observed, and at the poleward margin of the tropics, above the "horse latitudes, "the subtropical jet stream has been discovered.

In contrast, the equatorward branch near the surface is in contact with the ground. Under conservation of momentum, huge easterly winds should appear in the tropics. But, due to the boundary stress, momentum from west is transferred to the atmosphere, and the easterlies are held to the strength of the pleasant breezes of the trades (fig. 1).

With this reasoning, the observed distribution of east and west winds is deduced qualitatively from the meridional cell. The resulting vertical shear of the zonal winds is such that angular momentum is carried poleward, since the surface equatorward flow is associated with easterly winds and the poleward branch with westerly winds. 
The foregoing, in essence, summarizes the simple cell theory; it is a closely knit story which qualitatively, hangs well together. Mathematical models putting the chain of reasoning into quantitative form have been developed. Circulations as outlined in fig. 2 have been produced successfully in laboratory experiments on rotating fluids with equatorial heat and polar cold sources (Fultz, 1959). It would be very interesting now to investigate long period weather trends in middle latitudes -- month or longer -- with reference to the tropical cell. If we postulate variations in the intensity of the cell on this time scale, changes in heat and momentum export from the tropics would undoubtedly occur. But serious difficulties have come to light which lead us to inquire whether the entire simple cell model should not be summarily discarded.

\section{Objections to Simple Cell Model}

No one will insist that the assumptions, on which the model is based, hold rigidly. It is merely expected that effects due to departures from the assumptions are small relative to the effects due to the mean circulation. But there, precisely, doubt has arisen.

The assumptions break down strikingly near latitude $30^{\circ}$, where the tropical cell ends. How are heat and momentum transported there? An answer has been found for the northern hemisphere, based on the increased network of upper-air stations around the subtropical belt during the last 15 years. Balloons are sent up, usually twice daily, to measure pressure, temperature, humidity and wind over at least the lower $9 / 10$ of the atmosphere (by weight). These observations have shown that at least at the boundary of the tropics the flow is not steady, but "turbulent, " in horizontal planes. Trains of waves and 
vortices, with dimensions of the order of 1000 miles, produce alternating northerly and southerly wind components around the belt. The magnitude of these components is $10 \mathrm{mps}$ as compared with $1 \mathrm{mps}$ for the meridional cell. Now, in line with expectations from the source and sink locations, winds from south carry higher heat and momentum than winds from north. This correlation, though far from perfect, is sufficiently high to account for the bulk of poleward heat and momentum flow ( $c$ f Bjerknes, 1955). With this, turbulent transport by means of lateral eddies - - turbulent, because net mass flow is not considered -- replaces transport by means of correlations along the vertical in the simple cell, which does require net mass flow at any level.

The next question is: how important is the mechanism just described within the tropics proper. Near the surface the evidence is negative. Wind systems such as northeast trade and monsoon are among the steadiest on earth. During winter, equatorward flow takes place at almost all meridians. In the low levels, then, the meridional cell definitely denotes more than just the statistical average of all kinds of variable wind fluctuations. In the upper troposphere, however, large wind eddies and restlessness of flow occur often (fig. 3). Scanty evidence suggests that these eddies often extend across the equator. With this, several of the premises underlying the cell hypothesis are placed in jeopardy. Other evidence, mainly from precipitation data, also points to variability of weather elements within the tropics, though on varying time scales. India's food supply hangs in balance each year as the yield of the monsoon rains is awaited with anxiety. Even Honolulu, in the center of the trades, has experienced annual precipitation ranging from 10 to 47 inches in 70 years. Variability over shorter time intervals is still greater. 
Lateral turbulence, as depicted in fig. 4, transports momentum more effectively than heat; the correlation between north-south wind component and temperature decays rapidly from the subtropics equatorward. It will be difficult to eliminate poleward energy transport by the meridional circulation as a major factor on the heat budget, even though the flow of the return branch is not uniformly distributed around the tropics, but occurs with preference in a few narrow poleward-directed channels (Riehl 1950). In addition, an entirely different mode of heat transfer has lately attracted the attention of meteorologist and oceanographer: transport within the oceans. Consider a schematic ocean basin (fig. 4). In the broadest sense the surface water masses follow the imposed air circulation, clockwise in the northern hemisphere. This leads to poleward transport of warm water over the western parts of the oceans, and the equatorward transport of cool water from the north over the eastern parts; hence the shape of the isotherms in fig. 4. The existence of warm and cold currents has been known for a long time. But only recent calculations have suggested that as much as 25 per cent of the equatorial heat excess may be exported by these currents. It is tempting to speculate on the impact of variations in this heat flow. which may be brought about by changes in the intensity of the clockwise atmospheric circulation over the oceans. No doubt such variations must have an impact on atmospheric circulation anomalies of middle latitudes, hence become an important ingredient in long range weather prediction when the data necessary for computations become available.

In view of the foregoing we should, perhaps, carry investigations of the tropical general circulation forward mainly by computing turbulent 
fluxes, a task which will be difficult until the data density over the vast tropical area is greatly increased. Yet, this may be as exasgerated an approach to the circulation of the tropics as rigid adherence to the meridional cell. After all, the climatic scheme of subtropical deserts trade wind belt - equatorial rain zone does prevail and attest to the realism of the mean circulation scheme of fig. 2. This indisputable fact, together with reluctance to part with the mean cell hypothesis as a clear and possibly very sharp tool for gaining insight into the nature of the tropical circulation, has prompted further examination of the simple cell hypothesis.

Subtropical Jet Stream and Transverse Mass Circulation

As is well recognized (Bjerknes et al 1933), the principal difficulty of the cell hypothesis is the postulate of poleward moving rings of air which conserve their angular momentum. This postulate requires huge westerly wind speeds in the tropics and phenomenal speeds at higher latitudes. Actual profiles of zonal wind with latitude fall far short of the profile demanded by conservation of momentum, with the notable exception of East Asia (fig. 5). Bjerknes proposed to relax the requirement of poleward moving rings of air by taking advantage of the existence of subtropical high pressure centers in both northern and southern oceans. Consider the sketch of fig. 6 . Air moving northward at point $B$ is subjected to a westward directed pressure force. This force acts to decrease the angular momentum of the air at point $B$ which, then, can move northward without necessity of turning eastward with excessive speed. The converse applies at point $A$. In this way, the standing pattern of high pressure centers facilitates north-south exchange of air. 
With the increase of upper-air data in the 1950's, and especially with introduction of radar-type equipment to measure upper winds, the subtropical jet stream has emerged as a definite feature useful for delineating the poleward boundary of the tropics. During the winter of 1955-56, this current had the mean position and wavelike shape apparent in fig. 7. Broadly, this configuration recurs annually during winter. Fig. 7 clearly shows how nature has responded to the momentum problem. A strong subtropical velocity maximum exists at about the altitude of the core of the poleward branch of fig. 2. But this maximum undulates with longitude, and it is in phase with the pressure field so that angular momentum is abstracted during poleward flow and added during equatorward flow.

These observations suggest that it may be of value to examine some aspects of the tropical circulation in a curvilinear coordinate system following the waves of the subtropical jet stream, rather than in spherical polar coordinates which has been the usual practice. It is certainly desirable to have a latitude representation in any coordinate system, because the gradients of earth's momentum and of solar radiation lie in that direction. But the merits of the use of spherical coordinates as a means for describing and analyzing general circulations have not been investigated as such. Nothing need prevent us in principle from choosing reference frames, suitable for summarization of observational features, in any desired way.

Introduction of curvilinear coordinates following the subtropical jet stream was suggested by the use of such coordinates in a rotating dishpan experiment with three steady waves (Riehl and Fultz 1957, 1958). These waves may be treated by statistical methods as turbulence elements in a polar coordinate system, or they may be 
analyzed in curvilinear coordinates following the waves (fig. 8). When heat transfer is computed in both of these coordinate systems, the results are as follows. In polar coordinates, heat transfer is effected through the correlation of inward directed flow with high temperature, and vice versa (fig. 9). In curvilinear coordinates, it is produced by a cell similar to fig. 2, except that the mass flow is computed normal to the $\mathrm{s}$-axis of fig. 8 rather than normal to latitude circles.

This computation points out that, at least at the present stage of our thinking, characterization of a fluid -- at least of the steady state type -- is not absolute but dependent on the choice of reference system. Mathematically, the transports computed from all reference systems must, of course, agree. But it is evident that the success of a particular investigation maydepend critically on the reference frame used. In the case under discussion, the entire single cell model of the tropical circulation can be saved -- if that is desired -- by viewing the problem with curvilinear rather than spherical coordinates. Eventually, of course, the reasons for the observed position and wave structure of the subtropical jet streara must be understood. But lack of a complete solution at this time need not prevent our taking advantage of this structure for computations on the tropical circulation.

When the curvilinear reference frame relative to the subtropical jet stream is adopted -- with validity over a distance of at least 1000 miles equatorward from the jet axis - a mass circulation is obtained (Krishnamurti 1961) which, in large measure, accomplishes the heat and momentum transfer as postulated in the single cell model. Fuxther, a release of potential energy is computed which is about an order of magnitude greater than that required to maintain the motion against friction. This potential energy release 
is considerably larger than the production of kinetic energy by the meridional motion as computed by Palmén et al (1958). It actually has the magnitude which Pisharoti (cf. Bjerknes 1955) has estimated to be required for the maintenance of the whole extratropical circulation against friction. This shows clearly that the tropical wind systems are not driven by energy released by middle latitude disturbances -- as has been suggested -- but that the tropics make an important and, following Krishnamurti, also variable contribution toward maintaining the temperate zone winds.

A calculation showing the maintenance of the temperature structure equatorward of the subtropical jet stream may serve as an example of computations performed in the curvilinear system. In the sinking branch of the tropical cell (fig. 2) -- when referred to the curvilinear coordinates -- air is compressed because of motion toward higher pressure. This produces heating and tends to increase the temperature of the whole tropical atmosphere in the descending branch. For a steady temperature field, radiation cooling must balance this compression heating. If such balance is not achieved, at least roughly, then temperature advection by unsteady flow features must be an important factor in the circulation. As seen from fig. 11, balance is achieved within reasonable limits, and the computation may be taken as supporting the validity of the simple cell approach.

These calculations, plus others, demonstrate that the concept of a thermally induced mass circulation as the basis of the tropical general circulation may be upheld. The future will show whether this approach is rewarding as compared with the turbulence method, 
which will certainly be pursued, if only because it lends itself easily to computations on large computers. Some of the possibilities that may be explored with the cell model will be outlined later. At present let us ask merely whether a cell model will still serve for representation of the main circulation features when heat and momentum sources are separated. Opportunity for a test of such a situation arises during the northern hemisphere summer, when the center of the heat source shifts from the equatorial zone to the subtropics over the Asian and African continents and when, in addition, the heat source becomes elevated toward the middle of the troposphere (by weight) over the Asian mountain ranges and high plateaus. Analogy with fig. 2 suggests a cell with a structure as sketched in fig. 11 where, however, the heat source is now situated at the latitude of smallest earth's momentum. Studies of the Asian tropics, undertaken primarily by Koteswaram (1958), have confirmed this structure from the upper-air observations available since the middle of the 1950's. The analogy between summer and winter cell models can be carried very far. It also suggests that the precize location of momentum sources and sinks with respect to a heat source is not significant for the existence of vertical cells produced by heat sources.

\section{Vertical Heat Transfer}

Up to now we have spoken of the tropical heat source as selfevident, an assumption made frequently without qualification. A more detailed look, however, reveals quickly that in the determina tion of the exact ways in which the tropical atmosphere does become a heat source is a challenging research problem. 
In the tropics, as elsewhere, the atmosphere is a radiational cold source. Long wave radiation going to space greatly exceeds the direct absorption of solar short-wave radiation within the atmosphere. The real recipient of excess heat is the earth itself which in the tropics, as noted before, is covered about 80 per cent by water. It is the transfer of heat from ocean to air that establishes the atmospheric heat source. Contact heating of air particles at the interface produces some of this transfer. But the bulk is derived through evaporation of water from the oceans. This water is held in vapor form in the atmosphere, and its "latent heat" is eventually released through condensation during ascent in cumulus clouds.

According to current theory, the sensible heat flux is proportional to the temperature difference between sea and air, the surface wind speed, and a roughness parameter characteristic of the ocean surface. Evaporation is proportional to the vapor pressure difference between sea and air, wind speed and roughness parameter. It is assumed further, that the air temperature is not warmer than the ocean temperature, since this would strongly inhibit buoyant motions in the surface layer. If these hypotheses on exchange at the interface are correct, the heat transfer depends partly on atmospheric and partiy on oceanic properties. Clearly, a tropical atmospheric heat source does not exist a priori or automatically. The atmosphere itself exercises considerable control as to where and when strong or weak heat flux from sea to air will take place.

The mechanisms, by which the heat gained from the earth is distributed to the high atmosphere, present challenging problems, except possibly in the layer below cloud base, usually about 2, 000 feet high in the trades. There the atmosphere is sufficiently well 
mixed so that diffusion by eddies of relatively small, though variable size may be postulated. For upward transport beyond the cloud base, consider at first the ascent of air with surface properties typical of, say, the Caribbean Sea in summer. In the absence of mixing with the environment, the ascent of this air is given by the solid curve of fig. 12 . The break in temperature lapse rate at $930 \mathrm{mb}$ denotes the cloud base; from this point upward condensation occurs. The dashed curve shows a mean Caribbean atmosphere in summer, which is very similar to mean atmosphere computed over comparable parts of other oceans in summer. By and large the two curves of fig. 12 have the same shape; this observation has led to the general conclusion that the condensation process is the main factor in shaping the vertical temperature structure over the tropical oceans.

Let us now return to the circulation cell of fig. 2. In view of the steady state prescribed for the cell, particles rising from the surface in the equatorial zone actually should follow paths similar to those depicted by fig. 12 and establish the temperature structure of these paths as the climatically observed structure in the rising part of the cell. Until recently, it was generally assumed that such was the case. But diligent study of many thousands of balloon ascents has uncovered hardly any individual soundings which show this structure. One could fall back on the fact that in the equatorial zone, as elsewhere, ascent of air to high levels does not occur uniformly over the entire area -- although the concept of random convection was widely held at one time -- but that ascent is concentrated in weather distur bances which do not cover more than one tenth of the whole equatorial area. However, even soundings taken in such disturbances do not conform to the expected structure. Instead, the picture presented by 
fig. 13 occurs regularly. In this diagram, the total energy of the air has been expressed in heat units $(\mathrm{cal} / \mathrm{gm})$. The energy forms considered are: specific enthalpy, potential energy and latent heat energy. The straight-line ascent at constant energy content corresponds to the undilute ascent shown in fig. 12; the other curves give the mean structure of equatorial and trade wind zones. It follows that, in the equatorial zone, energy flows down-gradient in the lower part of the atmosphere and up-gradient at higher elevations.

This is a most unexpected situation for conditions at the heat source. If the circulation seen in fig. 2 is superposed on this mean structure, energy must be transported upward across, say, the $500-\mathrm{mb}$ surface at $79.5 \mathrm{cal} / \mathrm{gm}$ and exported poleward near $200 \mathrm{mb}$ at $82.0 \mathrm{cal} / \mathrm{gm}$. We are thus left with a deficit which is augmented by the radiational cold source within the upper atmosphere. Clearly, the cell concept fails to provide for heat balance here and must a least be amplified by an assumption of turbulent transport. Noting this, letus inquire how turbulence plus net mass transport can produce the required heat transport where, to compound the difficulty, the transport must be in part down-gradient and in part up-gradient. Before doing so, may I comment that problems of such complexity are common in meteorology. At times it has been said that progress in this field is slow. Perhaps the problem just outlined will explain just why things have not always advanced as speedily as one might wish.

The difficulty really is restricted to the layer above $600-500 \mathrm{mb}$, in which the total energy content of the air increases upward. Lower down, cumulus clouds abound over those portions of the tropical oceans where an active heat source exists. From the fundamental 
work of Stommel (1946) it has been clear that these clouds are an effective means of funneling heat upward and thus imparting it to the environment through mixing. In turn, this mixing reduces the buoyancy of the cumuli and, on the average, their tops are found near the layer of minimum energy content in fig. 13.

This observation, by itself, does not solve the very interesting question of why the mixing process described does not continue all the way to the top of the troposphere. At this stage of our knowledge, it is possible merely to postulate a mechanism which can establish the observed structure of the tropical atmosphere. Say, that a small fraction of the cumulus clouds becomes much larger in horizontal extent than most, with diameters of several $\mathrm{km}$ as compared with 100 's of metes's for the vast majority of the clouds. Then mixing with the environment at the edges of these larger clouds will require some time before environmental air can penetrate to the center of an updraft and reduce the buoyancy there. Hence, if the buoyancy acceleration is large enough to permit the air in the core to acquire speeds sufficient for escape to high levels before its buoyancy is eroded, then the energy content of this air upon arrival at the upper levels would be typical of the surface layer. Actually, the time needed by buoyant elements to travel from cloud base to the high troposphere is of the order of half an hour or less. This time scale is satisfactory for the mechanism just described and has been postulated as the means for bringing the whole equatorward mass flow to high levels (Riehl and Malkus 1958). The concentration of the poleward return current near 200-150 mb, i.e. near the top of the troposphere, supports this viewpoint. 
The foregoing hypothesis implies the existence of two effective heat source locations: one near the surface and one near the tropopause. Heat is transported upward through mixing by cumuli in the low layers, and transported downward by compression heating of turbulent elements from the high troposphere (fig. 14). The tropopause itself is in equilibrium with the surface; it has the same heat content and represents the highest altitude to which air can penetrate from the ground.

If the viewpoint just outlined holds, a few thousand large clouds in the tropics will suffice to act as heat and mass funnels connecting equatorward and poleward branches of the mass circulation cell. The area covered by such ascending shafts is likely to be no more than one per mille of the area of the equatorial zone in view of the strong vertical velocities in the large clouds. Therefore, the chance of balloon release into one of these updrafts is extremely small; moreover, releases often must be postponed during heavy rain at the release site. It is consequently evident why almost none of the many daily soundings show an atmospheric structure similar to that of the straight line ascent in fig. 13, so that the hypothesis is virtually without direct verification. This difficulty may be overcome now through cloud photography by satellite, when pictures are of sufficient quality to permit a count of the large clouds.

From the foregoing it might be inferred that these large clouds form by random selection from millions of small cumuli. This, indeed, was the viewpoint held about tropical convection during the early part of this century. However, ample evidence exists to demonstrate that these clouds form in zones of mass convergence associated with weather disturbances which, roughly, have the same dimension 
as those of higher latitudes. Further, some types of disturbances, though well-developed only in the high atmosphere, suppress cloud development almost to the ground even in the presence of a surface heat source. Where clouds do occur, they tend to be arranged in lines, usually parallel -- and more rarely normal -- to the shear of the horizontal wind with height, and parallel to the wind itself in the absence of pronounced shear. Wide clear areas are situated between these "cloud streets". Such grouping of clouds shows the value of laboratory experiments on convection cells carried out over many years; it should encourage continued experimentation. As yet, the occurrence of the streets is still problematic, but experiments may contribute toward a solution.

Quantitative work on atmospheric cloud streets in the tropics, from small cumuli to deep cloud bands in hurricanes, has been started. Notably Woods Hole Oceanographic Institution under the leadership of Dr. J. S. Malkus has made an extensive photographic survey in the middle of tropical Pacific where land influences are nil. In the past, such influences have cnly too often distorted the conclusions of observers. Fig. 15 shows one of the tracks flown by Mr. Claude Ronne, who carried out the experiment. Along the path shown, as along others, cloud systems were measured and found to be structured in close relation to the general air flow present, from the ground to 40,000 feet altitude, especially the latter. There was even an area several hundred miles wide west of Hawaii which was almost cloudless during one traverse, hardly what one would expect in the trades. The area of the equatorial "convergence zone" was observed to have a very variable cloud structure in time and space, in agreement with all qualitative observations of the last twenty years. 
In the future, photographic experiments logically will be coordinated with satellite photography. The combination should go far in establishing the visible structure and motions of the cumuli which effect the vertical heat transport, and in showing this structure in the framework of the broader-scale array of convective clouds.

The Next Decade

This article has attempted to show that there is no shortage of ideas which may be brought to bear on the subject of the general circulation in the tropics. A serious handicap, however, is lack of adequate observations to test hypotheses satisfactorily. The years from 1945 to 1960 may be regarded as the era of initial attempts to establish the processes of the tropical general circulation by computational means. If the next decade is to bring these attempts to fruition, a drastic change in the observational program must occur. The most encouraging feature of recent years has been improvement in quantity and quality of weather data from many tropical regions, especially with regard to upper air data. This hard core of basic and indispensable observations must be at least doubled if reliable calculations of tropical weather processes are to be performed.

As the reader may suspect, a substantial contribution to knowledge is expected from satellite observations, because of the nature of these data and because of satellite coverage of the oceans and large continental areas where routine inadequate weather observations are inadequate. The actual heat source of the earth-atmosphere system in the tropics has never been measured. It is computed by an intricate chain of calculations (cf. London 1957)' which contains many assumptions, especially about cloud cover. The satellites will make 
direct measurements of incident solar radiation, reflected solar radiation (albedo) and long wave radiation on a continuing basis. These measurements should yield information on albecio and tropical heat source for the whole tropical belt, for hemispheres, for continents and oceans; with respect to seasons and with respect to specific large atmospheric disturbances. Most intriguing, perhaps, is the possibility of variations of albedo and net heat source, say, from one January to the next, for the tropics as a whole and for specific regions. Such variations should have an impact on the tropical circulation cell. It may be that the intensity of this circulation will respond, possibly with substantial lag, to variations in net heat source. Weather and circulation anomalies in the temperate zone of one or both hemispheres may follow. But what is the origin of variations in net heat source? Changes in cloud cover are thought to be the most likely cause of variations of albedo. Since the atmospheric circulation itself controls the cloud cover, as far as is known, fluctuations in heat source may be internally produced. Then again, the atmospheric control may not be so perfect as assumed. Factors such as ascent to the surface of subsurface water masses in the oceans may play a substantial role in regulating cloudiness.

It is idle to carry speculations very far in matters where numerous variables exercise partial control of weather and also interact with one another. Two firm statements can be made. Information on the heat source as a function of time and space is imminent. But: this information, taken alone, will not solve the atmospheric problems. It must be analyzed in conjunction with adequate observations on the circulation of the troposphere, the poleward heat and momentum fluxes, and the air-sea energy and 
momentum exchange. Advances in ocean buoy design hold much promise for great strides in knowledge of the interface processes. While the buoy program may be pursued mainly by oceanographic and related interests, establishment of a far-flung buoy network over the oceans will also provide the surface exchange data requisite as input for atmospheric calculations.

The main residual difficulty is the prohibitive cost of establishing an adequate network of balloon sounding stations. At the present juncture, it is utopian to think in terms of the station density which is really needed. Instead, all possible alternatives must be explored, even though they may provide only partial data. Successful flights of balloons, floating with the wind at constant pressure over long tracks, have attracted much attention in recent years. The outlook for the operationally proven design is poor, since the balloon package is heavy and regarded as a hazard to aviation. But the balloon program has provided stimulus for an ingenious new design of light-weight, superpressure balloons (Lally 1959). As many as 2,000 such balloons are envisioned to circle the globe at one time at various altitudes; information from them would be gathered by orbiting satellites. Lally's sounding system contains the brightest present hope for extensive wind and temperature data from all parts of the tropics. Further, it may turn out that the balloons will shun certain areas and circulation systems, while congregating in others. This would provide direct evidence of the location of the highly important areas of horizontal divergence and convergence. In some areas and at some altitudes the balloons may cross the equator readily and indicate the extent of coupling between hemispheres. 
Still further, the data from the balloon flights may solve highly interesting questions, such as the following: is there a circumpolar belt with winds from west in the high troposphere oves the equator? Westerly winds have been measured at many locations. But at present we do not know whether these westerlies extend around the globe as in middle latitudes. Verification of the existence of an equatorial westerly belt would be an outstanding discovery, for its angular momentum would exceed the maximum angular romentum of the earth and hence could not be gained by contact with the ground. Can a dynamical model be devised to explain such a belt? Undoubtedly, a fundamental advance in knowledge of atmospheric dynamics should result from the impact of such challenging observations. And, since there is a belt of east ward propagating disturbances on the equator of the sun, this advance should also contribute to the understanding of solar dynamics. 